\title{
Tangible Around-Device Interaction Using Rotatory Gestures with a Magnetic Ring
}

\author{
Victor Cheung \\ Carleton University \\ Ottawa, Ontario, Canada \\ victor.cheung@carleton.ca
}

\author{
Audrey Girouard \\ Carleton University \\ Ottawa, Ontario, Canada \\ audrey.girouard@carleton.ca
}

\begin{abstract}
The majority of mobile applications use built-in touchscreens and/or accelerometers to provide direct ways for user inputs. Yet, the need to manipulate the device itself (e.g. touch, tilt) poses usability issues such as occlusion and inaccuracy. To address these issues, research proposed using the built-in magnetometer and magnets to facilitate around-device interactions. However, there is little evaluation in how this technique impacts performance and user experience beyond simple docking tasks. To fill this gap, we explored the mobile gameplay context by implementing an interface that uses rotatory gestures from a magnetic ring as input, and compared two control mappings (angular and linear) with touch and tilt in a usability study using a mobile game. We found that rotatory gestures with the ring, when mapped to angular controls, were on par with touch and superior over tilt, and engendered greater gameplay experience and sense of mapping. Based on our findings, we discuss implications of using this technique for gameplay, as well as other applications.
\end{abstract}

\section{CCS CONCEPTS}

- Human-centered computing $\rightarrow$ Interaction devices; Interaction design; Mobile devices.

\section{KEYWORDS}

Controller, magnetism, rotation, around-device interaction

ACM Reference Format:

Victor Cheung and Audrey Girouard. 2019. Tangible Around-Device Interaction Using Rotatory Gestures with a Magnetic Ring. In 21st

Permission to make digital or hard copies of all or part of this work for personal or classroom use is granted without fee provided that copies are not made or distributed for profit or commercial advantage and that copies bear this notice and the full citation on the first page. Copyrights for components of this work owned by others than the author(s) must be honored. Abstracting with credit is permitted. To copy otherwise, or republish, to post on servers or to redistribute to lists, requires prior specific permission and/or a fee. Request permissions from permissions@acm.org. MobileHCI '19, October 1-4, 2019, Taipei, Taiwan

(c) 2019 Copyright held by the owner/author(s). Publication rights licensed to ACM.

ACM ISBN 978-1-4503-6825-4/19/10 .. \$15.00

https://doi.org/10.1145/3338286.3340137
International Conference on Human-Computer Interaction with Mobile Devices and Services (MobileHCI '19), October 1-4, 2019, Taipei, Taiwan. ACM, New York, NY, USA, 8 pages. https://doi.org/10.1145/ 3338286.3340137

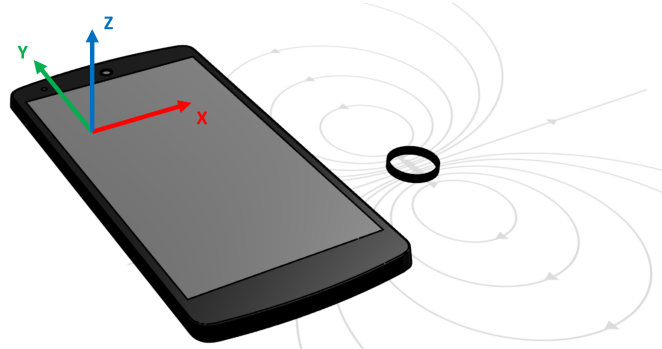

Figure 1: The coordinate system used by the mobile device's magnetometer to measure the strength of the surrounding magnetic field. Using this technique the magnetic ring becomes an input that requires no external power and placeable anywhere on the same $x y$-plane around the device.

\section{INTRODUCTION}

Modern mobile devices have a variety of sensor types embedded to expand their functionalities, from measuring surrounding ambient light (light sensor) to measuring atmospheric pressure (barometer). However, the input mechanism is still dominated by touch (and tilt, especially in games), which often suffers from usability issues such as occlusion, inaccuracy and overshooting $[12,24]$. One way to address these issues is to extend the interaction space around the device and provide users with tangible props [5, 13]. This approach both frees the display from occlusion and reintroduces tangibility and affordance of the controls.

Recent research has explored the use of magnetism as a low-cost and battery-free means for around-device interaction (ADI) $[5,7,13]$. By using the built-in magnetometers that are available in most current mobile devices, researchers have shown their potential to provide high resolution (microTeslas) and high frequency (typically over $60 \mathrm{~Hz}$ ) real-time inputs. Yet, the use of magnetometers is currently limited to utility applications such as compasses and metal detectors. Moreover, there is little work beyond proof-of-concepts (e.g. $[5,7,13])$ on how magnetism-based ADI impacts performance and user experience in mobile applications. 
In this paper, we explore the potential of using magnetometers to augment conventional input mechanisms. Through the use of a ring-shaped wearable magnet ${ }^{1}$, we made the "controller" easily carried around like a small accessory (Figure 1). Similar to recent research that used games as a vehicle to attract participants and provide a more realistic scenario (see [11, 19, 29] for examples), our study used a game to make the tasks more appealing and closer to the premise of a magnetic controller. To facilitate our exploration, we considered casual gameplay [32], which typically requires a small input vocabulary (e.g. swipes in Fruit Ninja [17]), as our initial application context. Our objective is to investigate how magnet-related input gestures can be mapped to ingame actions and how this mapping affects performance and user experience when compared to those using conventional sensors, namely, touch and tilt. We hypothesize that the tangibility and affordance of the external circular magnet would improve both, as reported by existing research in controller mapping [6, 20, 29], and tangible interactions [2].

Based on prior work using a ring for off-finger interactions $[7,8]$, we focus on the rotatory gestures (rotating the ring around its own axis on the same plane as the mobile device), which are easy to learn and perform, produce stable measurements and are unaffected by the ring's orientation (which side is up). This configuration also allows magnets in other shapes as input, as long as they can be rotated stably.

This paper makes two contributions. First, it is the first applying magnetism as the main input in a complete mobile game application. Second, it reports empirical results on performance and user experience between conventional and magnetic inputs, thus provides new and grounded insights to guide future research in using magnetism in ADI.

In the remainder of the paper, we discuss related work, implementation of the mappings, and our comparative study. Our results show that magnetic inputs lead to better performance and are most preferred when mapped properly. We conclude with design implications and potential applications.

\section{RELATED WORK}

We list prior research that has inspired us with the use of magnetism and built-in device sensors; as well as those that informed us with rotatory gestures and the relationship between naturalness of control action mappings and gameplay.

\section{Interacting with Small Devices using Magnets}

One advantage of using magnetism for input is the ability to interact outside the device, thus addressing usability issues with touchscreens (e.g. occlusion and precision [24]). As a form of Around-Device Interaction, researchers have used magnets to perform handwriting [9], item selection [3, 10],

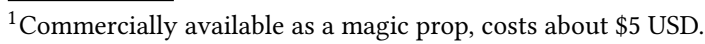

virtual object manipulation [14], and authenticate users [26]. Others have augmented physical objects, such as styluses $[1,36]$, GUI-components [13], or daily artifacts [5, 33, 37] to facilitate tangible interactions. However, these works remain in the research community and have not been adopted in any applications other than the proof-of-concept examples. This is likely due to the need for additional hardware, either to accurately track, or to house the magnet.

The Google Cardboard project [31] is perhaps the only magnetic setup that is commercially and widely available. By measuring the sliding motion of a magnet in a slot close to the device's magnetometer, a person can issue a press command, which, however, is the only input available.

In our recent work-in-progress abstract [7], we proposed rotating, lifting, and flipping a magnetic ring to enable additional controls, but did not conduct any study on the technique. We extend it by applying and evaluating the rotatory control to gameplay using two types of mapping (angular and linear), as will be described in later sections.

\section{Novel In-Game Mapping Using Built-in Sensors}

While not in the majority, there exist some games that use sensors other than the touchscreen, accelerometer, and gyroscope, or in an atypical way, in a mobile device to provide unconventional and novel ways to play.

Scream Go Hero [15] uses the microphone to pick up the player's voice as the input, and maps the loudness of the player's voice to the game character's movements (move and jump at various heights). Similarly, PlayableAle [4] maps the tone made by blowing across the top of a bottle to the game character's actions (dance and rage). Fast Like A Fox [18] uses the accelerometer to detect tapping motions of the player's fingers, and maps the speed of the taps to the game character's running speed.

Both Scream Go Hero and Fast Like A Fox are downloaded over 1 million times. We believe part of their popularity is due to their novel uses of the mobile device's sensors, and the appropriate mappings to in-game actions. In our study, we used game versions with controls that can be directly mapped to the rotatory gestures of the magnetic ring.

\section{Rotatory Gestures as Input}

Rotating a round object (dial/knob) has been a common input action in our daily lives (e.g. setting time/temperature [23]), and is incorporated into digital devices due to its familiarity. Voelker et al. [34] compared tangible rotary knobs and touchbased virtual knobs and found that the prior resulted in a faster control, and performance retained even when user's attention was elsewhere. Kim et al. [16] evaluated KnobSlider, which combines a knob and a slider in one unique shapechanging device, and reported that the knob shape allowed participants to precisely reach a parameter's value. 
These findings have shown that the use of a tangible rotary object and rotatory gestures provides a familiar and precise input mechanism, even without visual attention. We intend to investigate if the same applies in gameplay.

\section{Naturalness in Control and Gameplay Experience}

Research in gameplay has identified a positive correlation between naturalness in game control and gameplay experience (e.g. [21, 28, 30]). McEwan et al. [20] compared three controllers of various mapping naturalness (directional, incomplete tangible, realistic tangible) and discovered that a more naturally mapped device could lead to a higher degree of control, and provide greater potential for intuitive use. Shorey and Girouard [29] created a controller with a bendable midsection and mapped its deformations as gestures for in-game actions and navigation. The authors reported that simple actions that were naturally mapped to gestures tend to be preferred amongst participants, and were performed significantly better than more complex and abstract actions.

Much of the existing research has focused on the console gaming context, leaving whether the improvement in control and gameplay experience is transferable to the mobile gaming context an open research question. This work is intended to investigate such possibility.

\section{IMPLEMENTATION}

We describe how we used the magnetometer in a mobile device to measure the rotatory motion of a magnetic ring based on [7], and the two types of mappings we developed (angular and linear). For brevity, we describe our implementation using the terminology of Android API $23^{2}$, though an equivalent API is also available in iOS's Core Motion framework $^{3}$. Neither of these require any changes to the device or its operating system.

\section{Measuring the Rotatory Motion}

The measurements from a magnetometer are accessible via the Sensor.TYPE_MAGNETIC_FIELD flag passed to a SensorManager object. These measurements are in the unit of microTesla $(\mu \mathrm{T})$ and are along the $\mathrm{x}^{-}, \mathrm{y}^{-}$, and $\mathrm{z}$-axes relative to the device's coordinate system (Figure 1).

When a diametrically magnetized ring (the north and south poles are located at the curved surface, instead of the flat, circular surface, as illustrated in Figure 1) is rotated along the $\mathrm{z}$-axis of the device, the $\mathrm{x}$ - and $\mathrm{y}$-measurements change in a consistent manner, and return to the original value upon one full clockwise rotation (Figure 2).

Using this property, a simple arc-tangent trigonometric function can be applied to calculate an angular value $\theta$ :

\footnotetext{
${ }^{2}$ https://developer.android.com/guide/topics/sensors

${ }^{3}$ https://developer.apple.com/documentation/coremotion
}

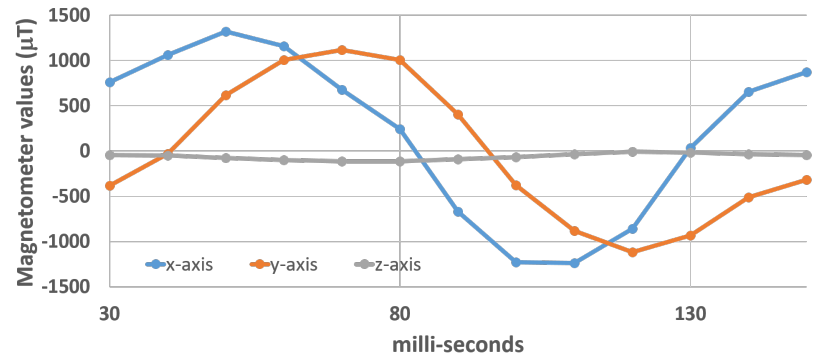

Figure 2: Magnetometer's measurements as a diametrically magnetized ring rotates one full circle (approx. $5 \mathrm{~cm}$ away).

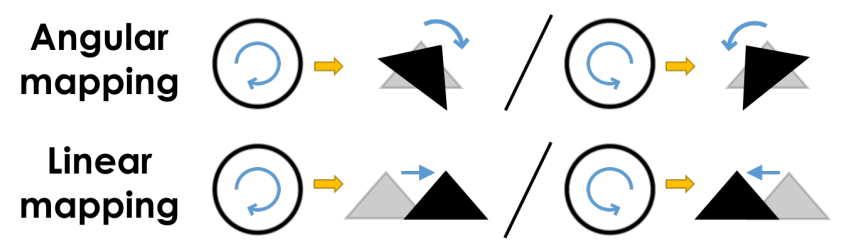

Figure 3: Illustrations of Angular and Linear in-game mapping. In angular mapping rotating the ring results in rotation of the game object; in linear mapping rotating the ring results in translation of the game object.

$$
\theta=\tan ^{-1}\left(\frac{y}{x}\right), \theta \in\left[-180^{\circ}, 180^{\circ}\right]
$$

We use this value as the input (in degrees) for the angular and linear mappings to generate in-app control.

\section{Two Types of In-Game Mapping: Angular and Linear}

The angular mapping is a straight-forward mapping directly adopting the value of $\theta$ described in the previous section to the rotational value of an in-game object or control, for example, heading of a projectile, or angle of a dial. On the other hand, the linear mapping uses the numerical value of $\theta$ as the input to an in-game object or control, for example, location of an item in one dimension, or value of a slider.

In both cases, if the value of $\theta$ is not modified, the mapping will also be continuous and ranged between -180 and 180 . Nevertheless, if required these properties can be changed using thresholds and scaling.

\section{USABILITY STUDY}

To understand how the use of magnetism in the form of rotatory motion impacts gameplay performance and experience, we conducted a comparative study between touch, tilt, and magnetism. We used touch and tilt because of their popularity in mobile games.

\section{Experimental Setup}

We selected Space Invaders [22] as the base design of the game we used in our study for the following reasons: 1) the gameplay is easy to understand and 2) the control is simple 


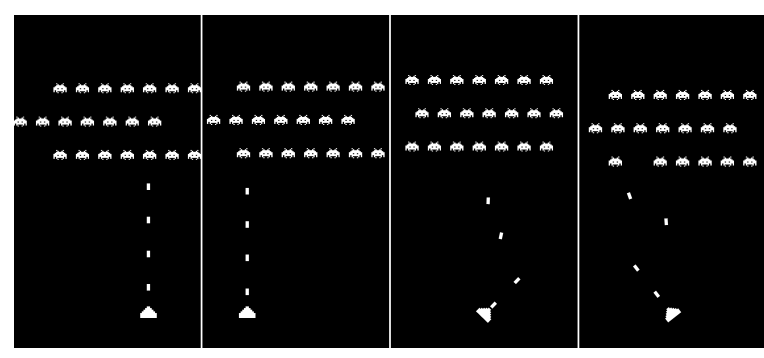

Figure 4: Screenshots of the game showing mappings to the canon's movements. In linear (left two), canon translates horizontally; in angular (right two), canon rotates.

and customizable to support all the input modalities in our study. In the original gameplay, the player moves a canon horizontally to shoot down descending aliens with lasers. The player wins by defeating all the aliens, and loses by not able to do so before they reach the bottom of the screen.

We made several modifications to the original gameplay to facilitate our study. The first two standardized the gameplay; whereas the second two facilitated comparisons between different input modalities and participants' skill levels:

- Smaller number of aliens so it takes less time to finish;

- Absence of elements such as defense bunkers and the "mysterious ship" to remove any randomization of game objects for consistent measurements across sessions;

- Inputs are for moving the canon only, which automatically shoots the lasers instead of requiring a separate input;

- Vertical movement of the aliens switches between upward and downward within $75 \%$ the screen, instead of always downward, so they are always in range and the player will always be able to defeat all of them without a time limit.

We implemented the game ${ }^{4}$ using Unity3D 2017, and installed it in an Nexus 5 mobile device running Android 6.0.1. Figure 4 shows screenshots with the two mapping schemes.

Game Inputs and Mapping Schemes. Based on the original idea of Space Invaders, we created three methods of input using touch, tilt, and magnetism (Figure 5). For each method, we created two mapping schemes for angular and linear in-game actions (Table 1), leading to six conditions in a 3 (touch/tilt/magnetism) x 2 (angular/ linear) factorial design.

In angular scheme, the canon rotates and shoots lasers at an angle; whereas in linear scheme, it translates horizontally and shoots lasers vertically. To turn/translate the canon, the player touches the left/right side of the screen, tilts the device, or rotates the magnetic ring correspondingly (Figure 3).

Study Measurements. We recorded the time required to finish one round of gameplay in each condition for objective measurements (shorter indicates better performance, also translates to accuracy as all aliens must be destroyed). We

\footnotetext{
${ }^{4}$ Source code available at https://github.com/thisisvictor/MagTroGames
}

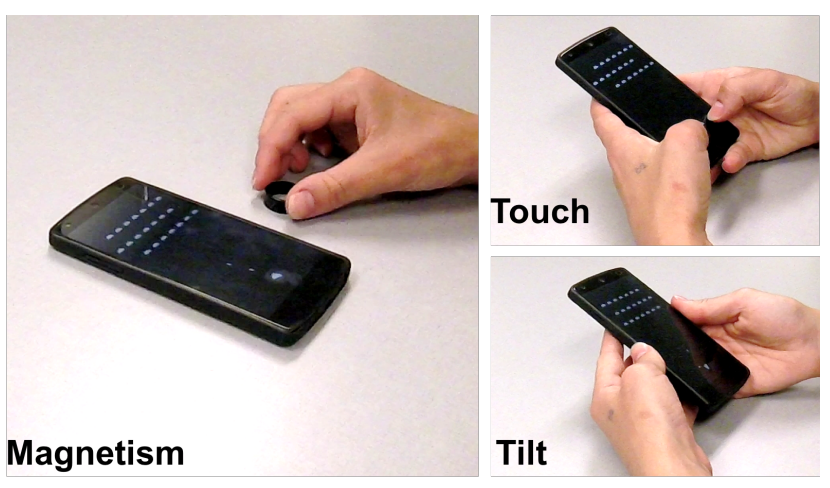

Figure 5: Three input methods (magnetism, touch, tilt) used in the study. Participants were free to hold or leave the phone on the table.

Table 1: The 3 input methods and 2 mapping schemes we created based on actions in Space Invaders.

\begin{tabular}{c|c|c}
\hline & Angular & Linear \\
\hline $\begin{array}{c}\text { Touch } \\
\text { (device) }\end{array}$ & $\begin{array}{c}\text { Touch left/right to } \\
\text { turn counter- } \\
\text { clockwise/clockwise }\end{array}$ & $\begin{array}{c}\text { Touch left/right to } \\
\text { move left/right }\end{array}$ \\
\hline $\begin{array}{c}\text { Tilt } \\
\text { (device) }\end{array}$ & $\begin{array}{c}\text { Tilt left/right to } \\
\text { turn counter- } \\
\text { clockwise/clockwise }\end{array}$ & $\begin{array}{c}\text { Tilt left/right to } \\
\text { move left/right }\end{array}$ \\
\hline $\begin{array}{c}\text { Magnetism } \\
\text { (ring) }\end{array}$ & $\begin{array}{c}\text { Rotate left/right to } \\
\text { turn counter- } \\
\text { clockwise/clockwise }\end{array}$ & $\begin{array}{c}\text { Rotate left/right to } \\
\text { move left/right }\end{array}$ \\
\hline
\end{tabular}

did not explicitly measure error rate, as "errors" in games could be ill-defined (e.g. shoot some lasers aimlessly just for fun, or have different strategies). We instead used how many times the lasers miss, which is indirectly represented by the completion time as the lasers are shot at a constant rate (shorter means less error). For subjective measurements, we employed the Short Version of the User Experience Questionnaire (UEQ-S) [27] to evaluate participants' user experience, and added the "Level of Naturalness" question used by McEwan et al. [20] to measure their impression on the naturalness of mapping inputs to in-game actions.

Procedures. The study session began with consent acquisition and a demographic questionnaire, followed by the participant playing a simplified version for each of the six conditions (three aliens instead of twenty-one) to familiarize themselves with the controls and gameplay.

When ready, the participant played the full version for each condition three times, each typically lasted one to two minutes. We employed a within-participant design where each participant experienced all six conditions in a randomized order, so as to minimize ordering effects. After each condition, we administered the subjective measurements as 
described in the previous section. At the end of the study session, we provided a post-session questionnaire to gather the overall preference and comments from our participants.

Each study session took approximately 60 minutes (up to 36 minutes of intermittent play time, along with training and questionnaires) to complete in an HCI laboratory. Each participant received $\$ 10$ for their time. The ethics research board of the institution approved this study.

\section{RESULTS}

We recruited 18 participants from our local community (6 male, average age: 25.8, S.D.: 4.5). All participants were righthanded and used their right hand to control the magnetic ring as input during the magnetism conditions. Figures 6 \& 7 show the measurements split by mapping schemes.

To analyze the data collected from our two-factor withinparticipant study design, we applied a two-way repeated measure analysis of variance (2-way RM-ANOVA) on the majority of the measurements. In the presence of interaction effect, we split the data into two subsets (Angular and Linear) for simple main effect analyses (1-way RM-ANOVA). To control for Type I error we used $\alpha=0.05 / 2=0.025$ (see [25]).

\section{Objective Measurements: Completion Time}

A 2-way RM-ANOVA on the average completion time for each of the six conditions (3 input methods and 2 mapping schemes) revealed significant differences between input methods $\left(F_{2,34}=21.88, \mathrm{p}<.001, \eta^{2}=.563\right)$ and mapping schemes $\left(F_{1,17}=70.10, \mathrm{p}<.001, \eta^{2}=.805\right)$, but also a significant interaction effect between the two factors $\left(F_{2,34}=35.03, \mathrm{p}<.001\right.$, $\left.\eta^{2}=.673\right)$. Upon splitting the data, our simple main effect analysis revealed a significant effect on the average completion time in both Angular $\left(F_{2,34}=31.07, \mathrm{p}<.001, \eta^{2}=.646\right)$ and Linear $\left(F_{2,34}=10.43, \mathrm{p}<.001, \eta^{2}=.380\right)$ schemes. A follow-up post-hoc test with Bonferroni correction in Angular scheme (Touch: 44.4s, Tilt: 59.0s, Magnetism: 42.1s) showed a significant difference between Touch \& Tilt $(\mathrm{p}<.025)$, Tilt \& Magnetism $(\mathrm{p}<.025)$, but not between Touch \& Magnetism. The same test on the Linear scheme (Touch: $31.4 \mathrm{~s}$, Tilt: $32.3 \mathrm{~s}$, Magnetism: 35.5s) showed a significant difference between Tilt \& Magnetism $(\mathrm{p}<.025)$, Touch \& Magnetism $(\mathrm{p}<.025)$, but not between Touch \& Tilt.

\section{Subjective Measurements}

$U E Q-S$. We followed the Short Version of User Experience Questionnaire (UEQ-S) analysis method [27], which revealed the pragmatic and hedonic qualities of the controls we tested. Table 2 summarizes the mean values for each of the six conditions reported by our participants. In UEQ-S, negative values represent undesirable qualities (e.g. obstructive, inefficient) and positive values represent desirable qualities (e.g. supportive, easy), within a range between -3 and 3 .

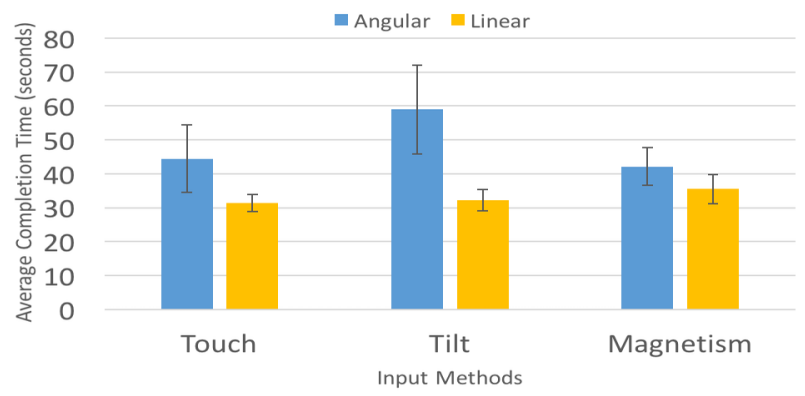

Figure 6: Average completion time for input methods split by mapping schemes, error bars show standard deviations.

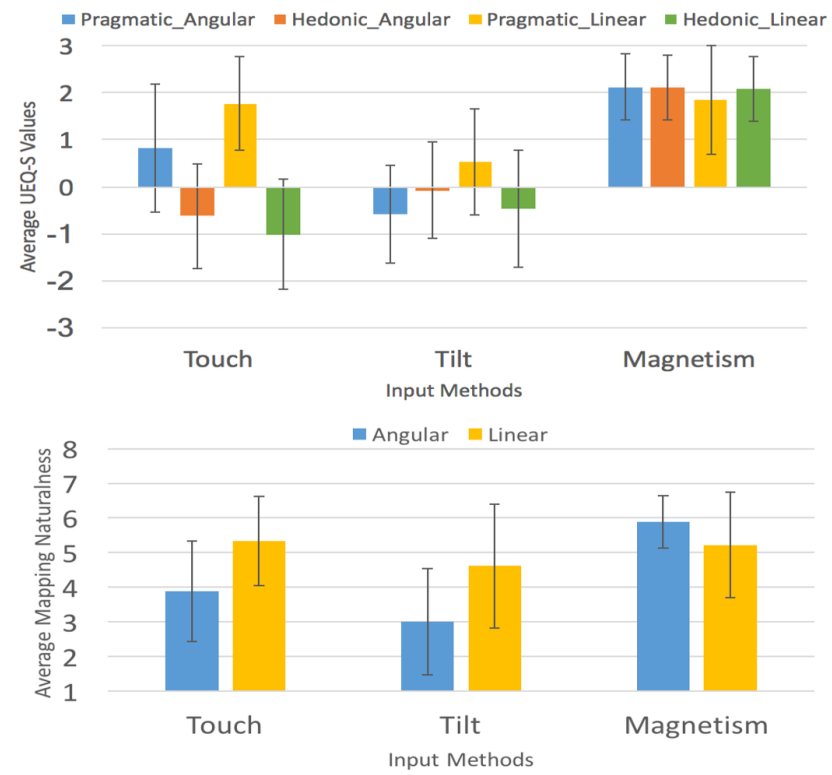

Figure 7: Average subjective values (top: UEQ-S, bottom: Mapping Naturalness) for input methods split by mapping schemes, error bars show standard deviations.

Table 2: Summary of UEQ-S results for the 3 input methods and 2 mapping schemes (range between $-3 \& 3$ ).

\begin{tabular}{|c|c|c|c|c|}
\hline & \multicolumn{2}{|c|}{ Pragmatic Quality } & \multicolumn{2}{c|}{ Hedonic Quality } \\
\cline { 2 - 5 } & Angular & Linear & Angular & Linear \\
\hline $\begin{array}{c}\text { Touch } \\
\text { (device) }\end{array}$ & 0.82 & 1.78 & -0.63 & -1.01 \\
\hline $\begin{array}{c}\text { Tilt } \\
\text { (device) }\end{array}$ & -0.58 & 0.53 & -0.08 & -0.47 \\
\hline $\begin{array}{c}\text { Magnetism } \\
\text { (ring) }\end{array}$ & 2.13 & 1.86 & 2.11 & 2.08 \\
\hline RM-ANOVA & $p<.001$ & $p<.001$ & \multicolumn{2}{|c|}{$p<.001$} \\
\hline
\end{tabular}

A 2-way RM-ANOVA on the average pragmatic quality rating revealed significant differences between input methods $\left(F_{2,34}=28.08, \mathrm{p}<.001, \eta^{2}=.623\right)$ and mapping schemes $\left(F_{1,17}=7.87, \mathrm{p}=.01, \eta^{2}=.317\right)$, but also an interaction effect between the two factors $\left(F_{2,34}=8.22, \mathrm{p}=.001, \eta^{2}=.326\right)$. A followup simple main effect analysis revealed a significant effect 
on both the Angular $\left(F_{2,34}=35.09, \mathrm{p}<.001, \eta^{2}=.673\right)$ and Linear $\left(F_{2,34}=9.73, \mathrm{p}<.001, \eta^{2}=.364\right)$ schemes. A post-hoc test with Bonferroni correction in Angular scheme showed a significant difference between all three input methods $(\mathrm{p}<.025)$; whereas the same test on the Linear scheme showed significant differences between Touch \& Tilt $(\mathrm{p}<.025)$ and Tilt \& Magnetism $(\mathrm{p}<.025)$, but not between Touch \& Magnetism.

On the other hand, in the absence of an interaction effect, a 2-way RM-ANOVA on the average hedonic quality revealed a significant effect with the input methods $\left(F_{2,34}=72.66, \mathrm{p}<.001\right.$, $\left.\eta^{2}=.810\right)$ but not with the mapping schemes $\left(F_{1,17}=3.24\right.$, n.s., $\left.\eta^{2}=.160\right)$. A follow-up post-hoc test with Bonferroni correction (Touch: -0.82, Tilt: -0.28, Magnetism: 2.10) showed a significant difference between Tilt \& Magnetism $(\mathrm{p}<.025)$, Touch \& Magnetism $(\mathrm{p}<.025)$, but not between Touch \& Tilt.

Level of Naturalness. We measured the level of naturalness using the same question in McEwan's study [20], giving us a Likert-scale value (from 1-Not natural at all to 7-Completely natural). We applied an AR transformation [35] on the 2factor non-parametric data and applied the same 2-way RM-ANOVA on the perceived naturalness of the six conditions. The analysis revealed significant differences between input methods $\left(F_{2,34}=8.43, \mathrm{p}<=.001\right)$ and mapping schemes $\left(F_{1,17}=26.93, \mathrm{p}<.001\right)$, but also an interaction effect between the two factors $\left(F_{2,34}=12.51, \mathrm{p}<.001\right)$. A follow-up simple main effect analysis revealed a significant effect on the Angular scheme $\left(F_{2,34}=26.49, \mathrm{p}<.001\right)$, where a post-hoc test with Bonferroni correction revealed a significant difference between Touch \& Magnetism $(\mathrm{p}<.001)$ and Tilt \& Magnetism $(\mathrm{p}<.001)$, but not between Touch \& Tilt. We did not find a significant effect in Linear scheme $\left(F_{2,34}=0.79\right.$, n.s. $)$.

Overall Preference. Lastly, we asked which input method our participants preferred the most in controlling the angle of the canon (Angular scheme), out of 18 of them, 3 preferred Touch for its "ease of control", none preferred Tilt, and 15 preferred Magnetism for it being "intuitive" and "interesting".

In controlling the canon's displacement (Linear scheme), 8 preferred Touch for its "ease of control" or "responsiveness", 2 preferred Tilt as it "simulates gravity", 7 preferred Magnetism as it was "interesting" and "innovative". One preferred Touch \& Magnetism for they were both "fairly responsive".

\section{DISCUSSION}

Summarizing our findings, we provide design implications using rotatory gestures with a magnetic ring.

\section{Use Rotatory Gestures for Angular Action}

Our results reveal that, when performing an angular action in the game, mapping the rotatory gestures of the magnetic ring to such action leads to the best performance (on par with touch, and better than tilt). Moreover, our participants rated it the best for pragmatic and hedonic qualities, and indicated overall preference on magnetism (15 out of 18).

We attribute the advantage of such mapping to the fact that the turning motion of a tangible round object corresponds to the turning action in the game ("incomplete tangible natural mapping" [30]). Also, we implemented a one-to-one mapping where the rate of rotation of the ring is the same as that of the game object, thus providing an "intuitive" sense of control.

When using a tangible object as control, designers should make sure that the in-game action reflects the gestures supported by the object. As doing so would improve performance, effectiveness and appeal [20, 21, 28-30].

\section{Using a Magnetic Ring for Mobile Devices as Input}

Rotating a round object for input is not a new idea (e.g. knobs/dials in various professional activities including sound engineering and aircraft piloting [16]). However, in most cases the knobs/dials are fixed to the device panel, and require a constant power supply. Using a magnetic ring for around-device interaction removes the need of external power, while addresses occlusion and accuracy issues. Moreover, the ring form factor of the magnet makes it easy to carry around as a jewelry item (or attached to any metallic items).

On the other hand, gestures supported by a magnet are limited, and while the rotatory gestures provide a responsive means of input, it could be affected by the surface the ring is on $[1,7]$. Also, the ring's magnetic field plays an important role in its optimal placement for sensing magnetic change, which explains why, while possible, we did not consider having the ring remained on the wearer's finger, as such placement would mean orienting the hands in a slightly awkward position and both occupied.

Nevertheless, our implementation has demonstrated the feasibility of interaction through magnetism. We expect the responsiveness and accuracy of this technique to be helpful in augmenting existing ones and beyond game controls, for example, making fine adjustments of a setting, scrolling quickly through a list, while touching the screen to confirm.

\section{Control Mapping Makes a Difference}

The presence of interaction effect in all but hedonic quality measurements strongly suggests that the choice of input method and in-game mapping affects each other, both objectively and subjectively. Splitting the data by mapping schemes (Angular and Linear) thus provides a better view of the impact on performance and user experience.

We observe better performance (average completion time) in all input methods with the Linear scheme. Participants also felt more effective, with more natural interactions with touch and tilt, as indicated by the higher ratings in pragmatic quality and level of naturalness. Yet, when mapped to angular actions, magnetism received a higher rating in both areas. 
On the other hand, the absence of interaction for hedonic quality could be due to the simplicity of the game, so participants did not find any of the mapping schemes particularly engaging. Nevertheless, our results showed a significantly higher hedonic quality score for magnetism, which many of the participants found it "fun", "natural", and "interesting".

When choosing a mapping scheme, designers must be aware of the relationship between the input mechanism and the in-app control. Using our implementation as an example, mapping the input methods to the linear movement of the canon generally results in a better performance, and the perception of effectiveness. Yet, mapping the magnetism input to the angular rotation of the canon has also led to those qualities. We elaborate more on this in the next section.

\section{Limitations}

The majority of our participants were university students who are familiar with touch/tilt on modern mobile devices. Recruiting a wider age and occupation range could provide variety in those skills and thus more comprehensive findings.

Regarding the study design, we only used variations of one game, which when replaced by another, could lead to a different outcome for the mapping schemes and other ways to map the touch and tilt inputs. Also, we only used the 8question UEQ-S instead of the full 26-question UEQ, which could have provided more insights on the subjective qualities.

While we acknowledge that the novelty effect might impact the subjective rating, we believe the naturalness of rotating a ring as input would have a lasting effect on the hedonic quality. We also believe the results will be sustained, given the pervasiveness of dials as a control, and the fact that the screen does not undergo any tilting or occlusion as compared to other forms of input. This, however, has to be verified by a longitudinal study and is out of the scope of this paper.

Nevertheless, we believe our study has revealed important aspects of the use of magnetism, and can serve as a good starting point for more research in this technique.

\section{CONCLUSION}

In this paper, we evaluated the impact on performance and user experience in a mobile gameplay context using rotatory gestures as control with an unpowered magnetic ring. We implemented and compared this technique with touch and tilt using a modified Space Invader game. We found that mapping the rotatory gestures naturally afforded by the ring's round shape to angular in-game action resulted in performance on par with touch, and superior over tilt. Our results also showed that people found such mapping effective and appealing, and preferred it over other forms of input.

We believe magnetic objects open up new and exciting possibilities in tangible around-device interactions with mobile devices due to their portability, cost-effectiveness, and freedom from external power. We hope our work will provide insights in further development of this technique and its applications beyond gameplay.

\section{ACKNOWLEDGMENTS}

This work was supported and funded by the Natural Sciences and Engineering Research Council of Canada (NSERC) through a Discovery grant (402494/2011), and the Collaborative Learning in Usability Experiences (CLUE) Create grant (465639-2015).

\section{REFERENCES}

[1] Tetsuya Abe, Buntarou Shizuki, and Jiro Tanaka. 2016. Input Techniques to the Surface Around a Smartphone Using a Magnet Attached on a Stylus. In Proceedings of the 2016 CHI Conference Extended Abstracts on Human Factors in Computing Systems (CHI EA '16). ACM, New York, NY, USA, 2395-2402. https://doi.org/10.1145/2851581.2892376

[2] Alissa N. Antle, Milena Droumeva, and Daniel Ha. 2009. Hands on What?: Comparing Children's Mouse-based and Tangible-based Interaction. In Proceedings of the 8th International Conference on Interaction Design and Children (IDC '09). ACM, New York, NY, USA, 80-88. https://doi.org/10.1145/1551788.1551803

[3] Daniel Ashbrook, Patrick Baudisch, and Sean White. 2011. Nenya: Subtle and Eyes-free Mobile Input with a Magnetically-tracked Finger Ring. In Proceedings of the SIGCHI Conference on Human Factors in Computing Systems (CHI '11). ACM, New York, NY, USA, 2043-2046. https://doi.org/10.1145/1978942.1979238

[4] Peter Bennett, Christopher Haworth, Gascia Ouzounian, and James Wheale. 2017. EchoSnap and PlayableAle: Exploring Audible Resonant Interaction. In Proceedings of the Eleventh International Conference on Tangible, Embedded, and Embodied Interaction (TEI '17). ACM, New York, NY, USA, 543-549. https://doi.org/10.1145/3024969.3025091

[5] Andrea Bianchi and Ian Oakley. 2013. Designing Tangible Magnetic Appcessories. In Proceedings of the 7th International Conference on Tangible, Embedded and Embodied Interaction (TEI '13). ACM, New York, NY, USA, 255-258. https://doi.org/10.1145/2460625.2460667

[6] Paul Cairns, Jing Li, Wendy Wang, and A. Imran Nordin. 2014. The Influence of Controllers on Immersion in Mobile Games. In Proceedings of the SIGCHI Conference on Human Factors in Computing Systems (CHI '14). ACM, New York, NY, USA, 371-380. https://doi.org/10.1145/ 2556288.2557345

[7] Victor Cheung and Audrey Girouard. 2018. Exploring Around-Device Tangible Interactions for Mobile Devices with a Magnetic Ring. In Proceedings of the Twelfth International Conference on Tangible, Embedded, and Embodied Interaction (TEI '18). ACM, New York, NY, USA, 108-114. https://doi.org/10.1145/3173225.3173283

[8] Ashley Colley, Virve Inget, Inka Rantala, and Jonna Häkkilä. 2017. Investigating Interaction with a Ring Form Factor. In Proceedings of the 16th International Conference on Mobile and Ubiquitous Multimedia (MUM '17). ACM, New York, NY, USA, 107-111. https://doi.org/10. $1145 / 3152832.3152870$

[9] Xinying Han, Hiroaki Seki, Yoshitsugu Kamiya, and Masatoshi Hikizu. 2007. Wearable handwriting input device using magnetic field. In SICE Annual Conference 2007. Takamatsu, Japan, 365-368. https: //doi.org/10.1109/SICE.2007.4421009

[10] Chris Harrison and Scott E. Hudson. 2009. Abracadabra: Wireless, High-precision, and Unpowered Finger Input for Very Small Mobile Devices. In Proceedings of the 22Nd Annual ACM Symposium on User Interface Software and Technology (UIST '09). ACM, New York, NY, USA, 121-124. https://doi.org/10.1145/1622176.1622199 
[11] Niels Henze, Enrico Rukzio, and Susanne Boll. 2011. 100,000,000 Taps: Analysis and Improvement of Touch Performance in the Large. In Proceedings of the 13th International Conference on Human Computer Interaction with Mobile Devices and Services (MobileHCI'11). ACM, New York, NY, USA, 133-142. https://doi.org/10.1145/2037373.2037395

[12] Ken Hinckley, Jeff Pierce, Mike Sinclair, and Eric Horvitz. 2000. Sensing Techniques for Mobile Interaction. In Proceedings of the 13th Annual ACM Symposium on User Interface Software and Technology (UIST '00). ACM, New York, NY, USA, 91-100. https://doi.org/10.1145/354401. 354417

[13] Sungjae Hwang, Myungwook Ahn, and Kwang-yun Wohn. 2013. MagGetz: Customizable Passive Tangible Controllers on and Around Conventional Mobile Devices. In Proceedings of the 26th Annual ACM Symposium on User Interface Software and Technology (UIST '13). ACM, New York, NY, USA, 411-416. https://doi.org/10.1145/2501988.2501991

[14] Hamed Ketabdar, Mehran Roshandel, and Kamer Ali Yüksel. 2010. Towards Using Embedded Magnetic Field Sensor for Around Mobile Device 3D Interaction. In Proceedings of the 12th International Conference on Human Computer Interaction with Mobile Devices and Services (MobileHCI '10). ACM, New York, NY, USA, 153-156. https: //doi.org/10.1145/1851600.1851626

[15] Ketchapp. 2017. Scream Hero. Game [Android, iOS]. Ubisoft Mobile, Bois, France.

[16] Hyunyoung Kim, Céline Coutrix, and Anne Roudaut. 2018. KnobSlider: Design of a Shape-Changing UI for Parameter Control. In Proceedings of the 2018 CHI Conference on Human Factors in Computing Systems (CHI '18). ACM, New York, NY, USA, Article 339, 13 pages. https: //doi.org/10.1145/3173574.3173913

[17] Halfbrick Studios Pty Ltd. 2010. Fruit Ninja. Game [Android, iOS] Taito, Tokyo, Japan.

[18] WayBefore Ltd. 2018. Fast Like a Fox. Game [Amazon, Android, iOS] Fingersoft, Oulu, Finland.

[19] Sven Mayer, Lars Lischke, Jens Emil Grønbæk, Zhanna Sarsenbayeva, Jonas Vogelsang, Paweł W. Woźniak, Niels Henze, and Giulio Jacucci. 2018. Pac-Many: Movement Behavior when Playing Collaborative and Competitive Games on Large Displays. In Proceedings of the $2018 \mathrm{CHI}$ Conference on Human Factors in Computing Systems (CHI '18). ACM, New York, NY, USA, Article 539, 10 pages. https://doi.org/10.1145/ 3173574.3174113

[20] Mitchell W. McEwan, Alethea L. Blackler, Daniel M. Johnson, and Peta A. Wyeth. 2014. Natural Mapping and Intuitive Interaction in Videogames. In Proceedings of the First ACM SIGCHI Annual Symposium on Computer-human Interaction in Play (CHI PLAY '14). ACM, New York, NY, USA, 191-200. https://doi.org/10.1145/2658537.2658541

[21] Rory McGloin and Marina Krcmar. 2011. The Impact of Controller Naturalness on Spatial Presence, Gamer Enjoyment, and Perceived Realism in a Tennis Simulation Video Game. Presence: Teleoperators Virtual Environments 20, 4 (Aug. 2011), 309-324. https://doi.org/10. 1162/PRES_a_00053

[22] Tomohiro Nishikado. 1978. Space Invaders. Game [Arcade, various] Taito, Tokyo, Japan.

[23] Geoff Norman. 2010. Likert scales, levels of measurement and the "laws" of statistics. Advances in health sciences education : theory and practice 15, 5 (10 Dec. 2010), 625-632. https://doi.org/10.1007/ s10459-010-9222-y

[24] R. L. Potter, L. J. Weldon, and B. Shneiderman. 1988. Improving the Accuracy of Touch Screens: An Experimental Evaluation of Three Strategies. In Proceedings of the SIGCHI Conference on Human Factors in Computing Systems (CHI '88). ACM, New York, NY, USA, 27-32. https://doi.org/10.1145/57167.57171

[25] John M Quick. 2011. R Tutorial Series: Two-Way ANOVA with Interactions and Simple Main Effects. Retrieved
May 20, 2019 from https://rtutorialseries.blogspot.com/2011/02/ r-tutorial-series-two-way-anova-with.html

[26] Alireza Sahami Shirazi, Peyman Moghadam, Hamed Ketabdar, and Albrecht Schmidt. 2012. Assessing the Vulnerability of Magnetic Gestural Authentication to Video-based Shoulder Surfing Attacks. In Proceedings of the SIGCHI Conference on Human Factors in Computing Systems (CHI '12). ACM, New York, NY, USA, 2045-2048. https://doi.org/10.1145/2207676.2208352

[27] Martin Schrepp, Andreas Hinderks, and JoİĹrg Thomaschewski. 2017. Design and Evaluation of a Short Version of the User Experience Questionnaire (UEQ-S). International fournal of Interactive Multimedia and Artificial Intelligence 4, 6 (12/2017 2017), 103-108. https://doi.org/ 10.9781/ijimai.2017.09.001

[28] Daniel M. Shafer, Corey P. Carbonara, and Lucy Popova. 2014. Controller Required? The Impact of Natural Mapping on Interactivity, Realism, Presence, and Enjoyment in Motion-based Video Games. Presence: Teleoperators Virtual Environments 23, 3 (Oct. 2014), 267-286. https://doi.org/10.1162/PRES_a_00193

[29] Paden Shorey and Audrey Girouard. 2017. Bendtroller: An Exploration of In-Game Action Mappings with a Deformable Game Controller. In Proceedings of the 2017 CHI Conference on Human Factors in Computing Systems (CHI '17). ACM, New York, NY, USA, 1447-1458. https://doi. org/10.1145/3025453.3025463

[30] Paul Skalski, Ron Tamborini, Ashleigh Shelton, Michael Buncher, and Pete Lindmark. 2011. Mapping the road to fun: Natural video game controllers, presence, and game enjoyment. New Media \& Society 13, 2 (2011), 224-242. https://doi.org/10.1177/1461444810370949

[31] Boris Smus and Christopher Riederer. 2015. Magnetic Input for Mobile Virtual Reality. In Proceedings of the 2015 ACM International Symposium on Wearable Computers (ISWC '15). ACM, New York, NY, USA, 43-44. https://doi.org/10.1145/2802083.2808395

[32] Gregory Trefry. 2010. Casual Game Design: Designing Play for the Gamer in ALL of Us. Taylor \& Francis.

[33] Rohan S. Vijay and Sidhant Goyal. 2016. MagnetoWear: A Magnetic Wearable Device to Interact With the Smartphone to Perform Personalized Actions. In Proceedings of the TEI '16: Tenth International Conference on Tangible, Embedded, and Embodied Interaction (TEI '16). ACM, New York, NY, USA, 597-602. https://doi.org/10.1145/2839462.2856554

[34] Simon Voelker, Kjell Ivar Øvergård, Chat Wacharamanotham, and Jan Borchers. 2015. Knobology Revisited: A Comparison of User Performance Between Tangible and Virtual Rotary Knobs. In Proceedings of the 2015 International Conference on Interactive Tabletops \& Surfaces (ITS '15). ACM, New York, NY, USA, 35-38. https: //doi.org/10.1145/2817721.2817725

[35] Jacob O. Wobbrock, Leah Findlater, Darren Gergle, and James J. Higgins. 2011. The Aligned Rank Transform for Nonparametric Factorial Analyses Using Only Anova Procedures. In Proceedings of the SIGCHI Conference on Human Factors in Computing Systems (CHI '11). ACM, New York, NY, USA, 143-146. https://doi.org/10.1145/1978942.1978963

[36] Sang Ho Yoon, Ke Huo, and Karthik Ramani. 2016. TMotion: Embedded 3D Mobile Input Using Magnetic Sensing Technique. In Proceedings of the TEI '16: Tenth International Conference on Tangible, Embedded, and Embodied Interaction (TEI '16). ACM, New York, NY, USA, 21-29. https://doi.org/10.1145/2839462.2839463

[37] Sang Ho Yoon, Yunbo Zhang, Ke Huo, and Karthik Ramani. 2016. TRing: Instant and Customizable Interactions with Objects Using an Embedded Magnet and a Finger-Worn Device. In Proceedings of the 29th Annual Symposium on User Interface Software and Technology (UIST '16). ACM, New York, NY, USA, 169-181. https://doi.org/10. $1145 / 2984511.2984529$ 